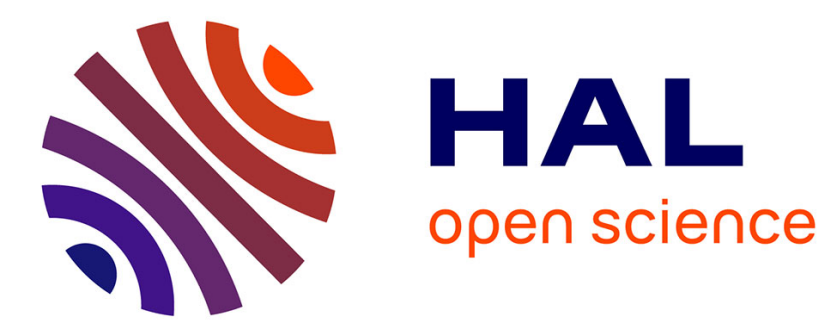

\title{
Travailleuses domestiques et autres stéréotypes sur les femmes migrantes
}

\author{
Speranta Dumitru
}

\section{To cite this version:}

Speranta Dumitru. Travailleuses domestiques et autres stéréotypes sur les femmes migrantes. L'Économie politique, 2019, 10.3917/leco.084.0059 . hal-02376335

\section{HAL Id: hal-02376335 \\ https://hal.science/hal-02376335}

Submitted on 22 Nov 2019

HAL is a multi-disciplinary open access archive for the deposit and dissemination of scientific research documents, whether they are published or not. The documents may come from teaching and research institutions in France or abroad, or from public or private research centers.
L'archive ouverte pluridisciplinaire HAL, est destinée au dépôt et à la diffusion de documents scientifiques de niveau recherche, publiés ou non, émanant des établissements d'enseignement et de recherche français ou étrangers, des laboratoires publics ou privés. 
Dumitru, S. 2019. Travailleuses domestiques et autres stéréotypes sur les femmes migrantes, L'Economie Politique, 84(4): 59-71

\section{Travailleuses domestiques et autres stéréotypes sur les femmes migrantes}

Durant de nombreuses décennies, les recherches sur la migration se sont intéressées surtout aux hommes. Les statistiques montraient que les femmes migraient aussi, mais les chercheurs supposaient qu'elles ne faisaient qu'accompagner les hommes comme épouses ou comme filles. Puis, à la fin des années 1980, on commence à évoquer une «féminisation de la migration » : les femmes seraient désormais majoritaires dans la population migrante ou du moins, leur part augmenterait de manière régulière et substantielle. De plus en plus de recherches rappellent la «féminisation de la migration » et l'associe aux emplois genrés, notamment au travail domestique. En une décennie, l'expression «féminisation de la migration» est entrée dans les dictionnaires. On peut y lire par exemple que « la féminisation de la migration produit des formes genrées de migration, en particulier en termes de travailleuses domestiques ou de care fourni à domicile ou dans les maisons de santé ; le trafic des femmes pour lindustrie du sexe et la migration organisée pour mariage » (Rothschild, 2009). Et si les femmes étaient sorties de l'invisibilité pour entrer dans les stéréotypes?

Cet article explore le stéréotype qui associe la migration des femmes au travail domestique, en analysant ses conditions de production. L'un des mécanismes psychologiques est la " cohérence associative », c'est-à-dire notre reflexe à construire une histoire cohérente en associant des informations disponibles mais incomplètes. Pour le saisir, prenons l'exemple de la théorie du "care drain », élaborée par la sociologue des émotions, Arlie Hochschild. Après avoir mené des entretiens avec des nounous immigrées aux Etats-Unis, Hochschild (2004) en conclut que la migration des femmes reflète " une tendance mondiale d'une ampleur croissante : l'importation de soins et d'amour des pays pauvres vers les pays riches ». Elle y voit le signe d'un nouvel impérialisme qui n'opère plus une extraction de matières premières mais s'étend aux "ressources émotionnelles ». A première vue, il s'agit seulement d'une généralisation hâtive. Mais Hochschild propose une histoire cohérente et plausible sur les femmes comme "chaînes mondiales de care » : les femmes migrantes prennent soin des enfants des femmes des pays riches et envoient de l'argent à d'autres femmes pour prendre soin de leurs propres enfants laissés dans les pays pauvres. Au bout de chaque " chaîne mondiale de care », il doit y avoir un " care drain ", c'est-à-dire une perte de soins et d'amour pour les enfants des pays pauvres.

Pour qui est sensible aux inégalités mondiales, ce récit est convaincant. Mais les migrantes travailleuses domestiques dans les pays riches, en Europe et Amérique du Nord, représentent moins de $2 \%$ de l'ensemble des femmes migrantes : comment pourraient-elles refléter une tendance mondiale? Nous avons trop vite cédé aux sirènes du stéréotype. 
L'objectif de cet article est d'offrir des outils pour éviter les stéréotypes, en prenant appui sur les concepts de psychologie cognitive ${ }^{1}$. Comment notre reflexe d'associer des informations limitées en une histoire cohérente peut-elle biaiser nos jugements?

L'article est structuré en trois sections. La première section présente l'évolution des stocks des migrants internationaux par sexe, pour montrer que les données disponibles ne justifient pas les discours sur la « féminisation de la migration ». Mais pourquoi alors cette expression est-elle devenue populaire ? En analysant ses usages dans la littérature scientifique, on découvre qu'elle est liée à une théorie de la mondialisation qui la prédit. C'est la théorie de la "nouvelle division du travail internationale et genrée » et elle sera présentée dans la deuxième section. Or, des théories compréhensives - comme celle-ci, qui va de la mondialisation et la mobilité du capital à la migration des femmes - sont susceptibles d'être biaisées. La troisième section montre donc comment la cohérence associative active la pensée par stéréotype.

\section{Féminisation ou masculinisation de la migration?}

Le succès de l'expression " féminisation de la migration » est déroutant compte tenu des statistiques migratoires disponibles. La formule est devenue si populaire qu'elle figure dans des dictionnaires et encyclopédies. Dans la littérature qui emploie cette expression, on soutient l'une des trois opinions suivantes : i) le nombre absolu des femmes migrantes a augmenté ; ii) les femmes migrantes représenteraient la majorité des migrants internationaux; iii) les femmes représenteraient une proportion croissante dans la migration internationale.

Or, pour chacune de ces significations - croissance en nombre, en proportion, majorité - les statistiques migratoires suggèrent qu'il serait plus judicieux de parler de « masculinisation » plutôt que de «féminisation » de la migration internationale. Regardons les données de la Division Population des Nations Unies (cf. Tableau 1).

Premièrement, si par «féminisation » on entend l'augmentation du nombre de femmes migrantes, les données montrent qu'entre 1960 et 2017, ce nombre a augmenté de 36 à 124 millions, c'est-à-dire de 88 millions. Mais le nombre d'hommes migrants a augmenté de 93 millions, en passant de 40 à 133 millions pendant cette même période. En volume, il y a donc eu une « masculinisation de la migration » - plus marquée, mais moins remarquée - que la « féminisation de la migration ».

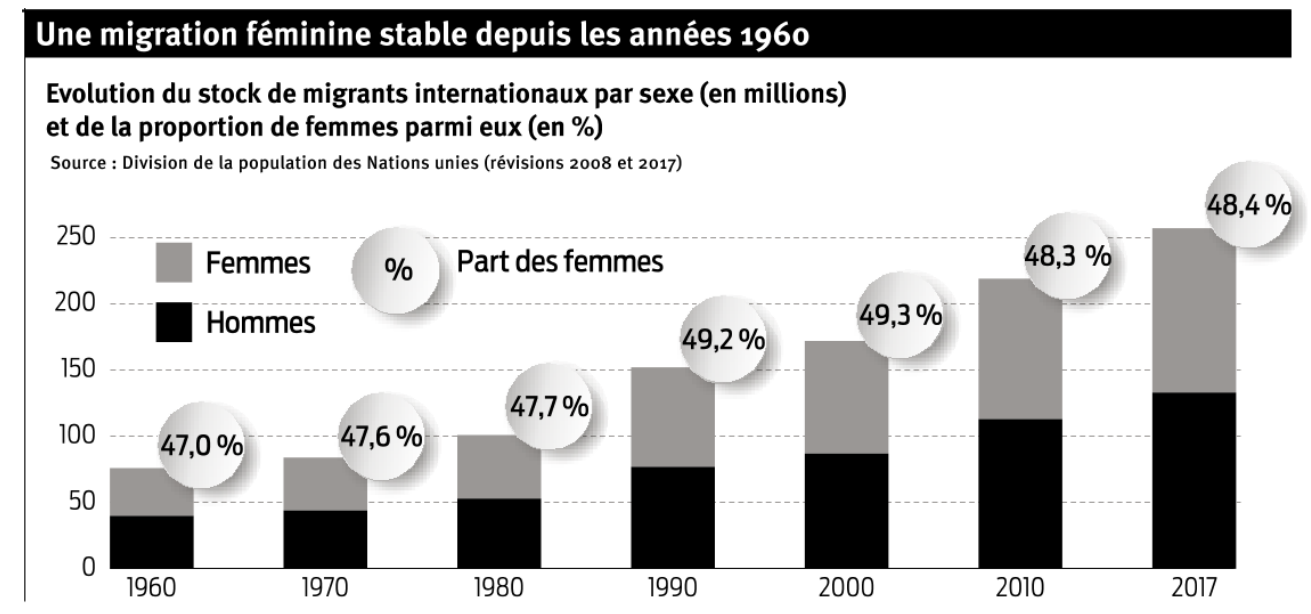

\footnotetext{
${ }^{1}$ Kahneman (2012) offre une excellente entrée dans la matière.
} 
Deuxièmement, si par « féminisation de la migration » on entend dire que les femmes seraient devenues majoritaires dans la migration internationale, les données des Nations Unies ne confirment pas l'existence d'un tel phénomène. Durant plus d'un demi-siècle, les hommes sont restés majoritaires, sans que l'on évoque, sauf exception, la « masculinisation » de la migration internationale.

Enfin, si par «féminisation » on entend une augmentation de la proportion des femmes dans la migration internationale, on observe que cette augmentation a été très faible au cours d'un demi-siècle, passant de $47 \%$ en 1960 à 48,4\% en 2017. Faut-il parler de «déféminisation de la migration », lorsque cette proportion baisse après 2000 ?

Le succès de l'expression « féminisation de la migration » est d'autant plus déroutant qu'elle est devenue populaire en dépit de la publication des statistiques des migrations internationales. Les premières estimations des stocks des migrants internationaux sont publiées en 1998 et montrent que la proportion des femmes est restée stable depuis 1960. Ce n'était pas une surprise car Hania Zlotnik, alors directrice de la Division Population des Nations Unies, l'avait déjà annoncé quelques années auparavant (Zlotnik, 1990).

L'exemple le plus déroutant est celui du sociologue Stephen Castles qui participe en 1998 à un Symposion Technique des Nations Unies sur la Migration internationale et le développement et publie un rapport de conférence qui précise que "la notion de féminisation de la migration ne peut pas être justifiée en termes purement quantitatifs » (Castles, 1998). Mais les éditions suivantes de son livre caractérisent la " féminisation de la migration » comme l'une des cinq principales tendances de la migration internationale, en mentionnant que les femmes seraient majoritaires dans certains corridors migratoires (Castles et Miller, 2003). Or, en 2000, les pays avec la proportion la plus élevée de femmes parmi les immigrés sont le Népal (66\%), Maurice (63\%), Estonie (59\%), Pologne (59\%), Arménie (58\%), ainsi que de nombreuses petits états, notamment de l'ancienne Union Soviétique. Du point de vue des statistiques migratoires, l'éclatement de l'Union Soviétique en 1990 a eu pour effet une augmentation artificielle du stock de migrants, car les personnes considérées auparavant comme des migrants internes ont été reclassifiées comme migrants internationaux, sans qu'elles aient traversé de frontière.

Plus étonnant encore: le succès de l'expression «féminisation de la migration internationale » non seulement n'a pas tari après la publication des données statistiques, mais s'est amplifié au cours des années 2000. Un nombre croissant d'études sur les femmes migrantes utilisent cette expression dans le titre même des articles : "La féminisation de la migration de travail comme violence contre les femmes » (Piper, 2003), "La féminisation de la migration : le care et le nouvel impérialisme émotionnel » (Gündüz, 2013), " La féminisation de la migration et le trafic des femmes » (Acharya, 2010). Comment expliquer un tel engouement ?

\section{De la circulation des capitaux à la migration des femmes ?}

L'une des explications de la persistance de la croyance en la « féminisation de la migration » (en dépit des statistiques qui la contredisent) peut être trouvée en analysant les usages de cette expression dans la littérature. Par exemple, dans une encyclopédie prestigieuse, on affirme que « la féminisation de la migration a été mise en évidence depuis 1980 lors de la période de mondialisation économique rapide » 
(Yoshimura, 2007). Ailleurs, on soutient que « les sciences sociales ont mis en évidence une croissance marquée du nombre de femmes migrantes, ainsi que de leur proportion dans le nombre total des migrants » et que "ce phénomène est lié à la transformation économique, politique, culturelle du capitalisme tardif » (Passerini et al., 2010).

S'il n'existe pas de féminisation de la migration dans les années 1980 (voir tableau 1), il existe, durant cette décennie, une théorie qui la prédit. C'est la théorie de la "nouvelle division internationale du travail » qui soutient que la circulation internationale des capitaux produit, entre autres conséquences, une « féminisation de la migration », les femmes migrant des pays en développement pour travailler dans les emplois subalternes des pays développés.

La théorie de la nouvelle division internationale de travail illustre le mécanisme de cohérence associative : à partir d'informations nécessairement limitées, on construit une théorie sur la " mondialisation ». Le premier ouvrage qui la théorise s'appuie sur une enquête menée auprès des entreprises allemandes (Fröbel et al, 1980). La délocalisation de certaines de leurs activités productives vers les pays en développement y est interprétée comme le signe d'une « restructuration économique mondiale ». On y soutient que l'expansion capitaliste crée une "nouvelle division du travail » entre les pays anciennement colonisés qui s'industrialisent et les pays occidentaux qui se spécialisent dans les services. Dans ce livre, les migrations SudNord, caractérisées avec l'expression de Marx comme "armée de réserve des travailleurs », sont expliquées comme le produit de l'expansion capitaliste.

La dimension genrée de cette théorie est élaborée par Saskia Sassen. Dans un livre sur la Mobilité du travail et du capital, Sassen (1988) soutient qu'il existe un lien causal entre la mobilité du capital et la migration des femmes. Pour elle, la division du travail s'opère entre les zones industrielles d'exportation des pays en développement et les " villes mondiales » des pays développés. Les deux types de lieux attirent la migration des femmes, à chaque fois vers les emplois peu qualifiés. Il y a d'abord, une migration interne: les zones industrielles "déracinent» les femmes du milieu rural et contribuent au «bouleversement des structures d'emploi traditionnel, souvent nonrémunéré ». Sassen semble déplorer le "recrutement massif de jeunes femmes (...) lesquelles ne seraient pas autrement entrées dans l'emploi rémunéré (...) [un phénomène] dont on a montré qu'il contribue au chômage des hommes et, dans certains cas, à leur émigration ». Il y a ensuite, la migration internationale : les zones d'exportation prépareraient les femmes à l'émigration en les "occidentalisant ». La « féminisation de l'emploi » serait suivie par la «pratique répandue du licenciement de nouveaux travailleurs, des jeunes femmes pour la plupart » lesquelles « devenues sans emploi et occidentalisées n'ont d'autre choix que d'émigrer ». Dans les villes mondiales, où la structure occupationnelle est supposée polarisée entre emplois hautement rémunérés et occupations mal-payées, les immigrés vont " servir les styles de vie des professionnels à hauts revenus " (supposés être non-immigrés). Pour Sassen, comme pour d'autres néo-Marxistes, la migration n'est qu'un produit de l'expansion capitaliste dont les conséquences sont néfastes pour les travailleurs locaux : "lïmmigration et la délocalisation sont des moyens de préserver une force de travail bon marché et de lutter contre les revendications des travailleurs organisés des pays développés ».

Depuis les années 1980, la théorie de la « restructuration économique mondiale » inspire de nombreuses études qui rappellent le lien entre mondialisation capitaliste et " féminisation de la migration ». Vu sous cet angle, l'article d'encyclopédie qui affirme 
que "la féminisation de la migration a été mise en évidence depuis 198o lors de la période de mondialisation économique rapide » n'est pas totalement faux : il existe bel et bien une théorie qui prédit la féminisation. Seulement, les statistiques sur la migration des femmes ne confirment pas les attentes de cette théorie.

\section{Trois raisons de se méfier des histaires plausibles}

La cohérence associative, cette disposition à construire une histoire cohérente en associant des informations, souvent incomplètes, peut biaiser nos jugements de plusieurs façons. Ici, on peut en mentionner trois: l'erreur de conjonction, la négligence des taux de base et le biais de confirmation.

Premièrement, l'erreur de conjonction est notre tendance à surestimer la probabilité d'une conjonction d'éléments surtout quand leur association nous semble plausible. Par exemple, la théorie de la division internationale du travail présuppose une triple chaine causale : i) l'investissement direct étranger détermine le recrutement massif des femmes dans les pays du Sud (féminisation de l'emploi), ii) lequel favorise leur migration vers les pays du Nord (féminisation de la migration), iii) où les femmes vont occuper des emplois peu qualifiés, mal rémunérés ou genrés (division internationale et genrée du travail).

Cette triple chaine causale rend la théorie plus plausible, mais moins probable. La raison en est logique : la probabilité d'une conjonction d'évènements est plus faible que la probabilité de chaque évènement pris isolément. De surcroît, chaque lien causal de la théorie fait l'objet de contestations. La question de savoir si l'investissement direct étranger a un impact sur l'émigration et dans l'affirmative, si cet impact est négatif ou positif est une question encore débattue aujourd'hui. La question de savoir si la migration interne est une condition nécessaire et suffisante de la migration internationale est également contestée.

L'erreur de conjonction peut être mieux comprise au moyen de l'une des expériences qui l'a mise en évidence : « le problème de Linda ${ }^{2}$. On décrit Linda comme une jeune femme brillante intéressée par les questions de justice sociale et on demande aux sujets ce qui est plus probable: que Linda soit «employée de banque » ou qu'elle soit « employée de banque et militante féministe ». La majorité des répondants choisissent la seconde option, oubliant que la conjonction (employée de banque et militante féministe) est une partie de l'ensemble, plus large, des « employées de banque ». Le fait que le profil de Linda soit construit comme un stéréotype de féministe biaise notre jugement. Au lieu de comparer la probabilité des deux options, on ne peut pas s'empêcher de voir Linda comme représentative du stéréotype.

L'une des raisons de se méfier des histoires plausibles est qu'elles peuvent soit propager soit activer des stéréotypes. La théorie du «care drain », citée en introduction, est un cas d'école de l'erreur de conjonction : on suppose que les migrantes qui sont « travailleuses domestiques et mères et avec des enfants laissés au pays d'origine » reflètent une tendance mondiale. Or, en ajoutant des critères, on restreint le nombre de personnes qui les remplissent : elles sont certainement moins nombreuses que les migrantes « travailleuses domestiques » tout court. Dans la figure 1, la conjonction (première case grise) nous semble plus plausible mais elle nous détourne de notre objectif. Si l'objectif est d'étudier les travailleuses domestiques (et

\footnotetext{
${ }^{2}$ Le problème de Linda, mis en evidence en 1983, peut être retrouvé dans Kahneman (2012)
} 
éventuellement, d'illustrer une division internationale du travail), leur catégorie comprend aussi des femmes sans enfant ou qui résident avec leurs enfants (cf. grand carré). Si l'objectif est d'étudier les enfants laissés au pays par leurs mères (pour explorer le care drain), il faut s’intéresser à toutes les mères migrantes, quel que soit leur emploi (cf. les deux cases grises). Or, le triple stéréotype (travailleuse domestique, mère et enfant au pays d'origine) pousse Hochschild à surestimer l'importance numérique d'une catégorie de femmes, en l'érigeant en «tendance mondiale d'une ampleur croissante ».

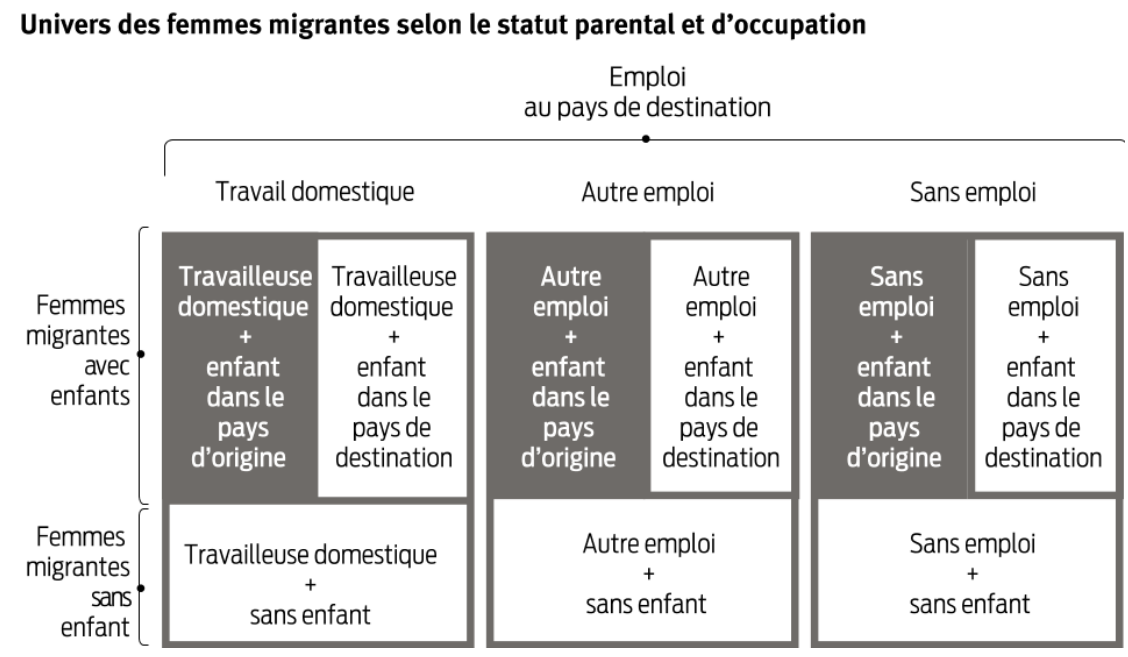

La deuxième raison de se méfier des histoires plausibles est qu'elles font oublier les taux de base, c'est-à-dire le poids relatif des populations impliquées dans la narration. Par exemple, le nombre de femmes migrantes employées comme travailleuses domestiques est de 8,5 millions 3 , soit $7 \%$ des femmes migrantes (voir fig. 2). Avec une telle part, il est difficile de caractériser « la féminisation de la migration » par le travail domestique. Mais il est encore plus difficile de considérer que la « féminisation de la migration » illustre une division entre les pays du Nord et du Sud car les migrantes qui sont travailleuses domestiques dans les pays riches de l'Europe et Amérique du Nord représentent $2 \%$ de l'ensemble des migrantes dans le monde. Même si toutes étaient originaires des pays du Sud et toutes avaient laissé leurs enfants au pays d'origine, il est clair que le «care drain » qu'elles produiraient ne reflète ni la migration des femmes, ni une division du travail entre le Nord et le Sud.

\footnotetext{
${ }^{3}$ Voir OIT (2015). Le calcul des proportions utilise les données des Nations Unies de 2013, comme le fait ce Rapport de l'OIT.
} 


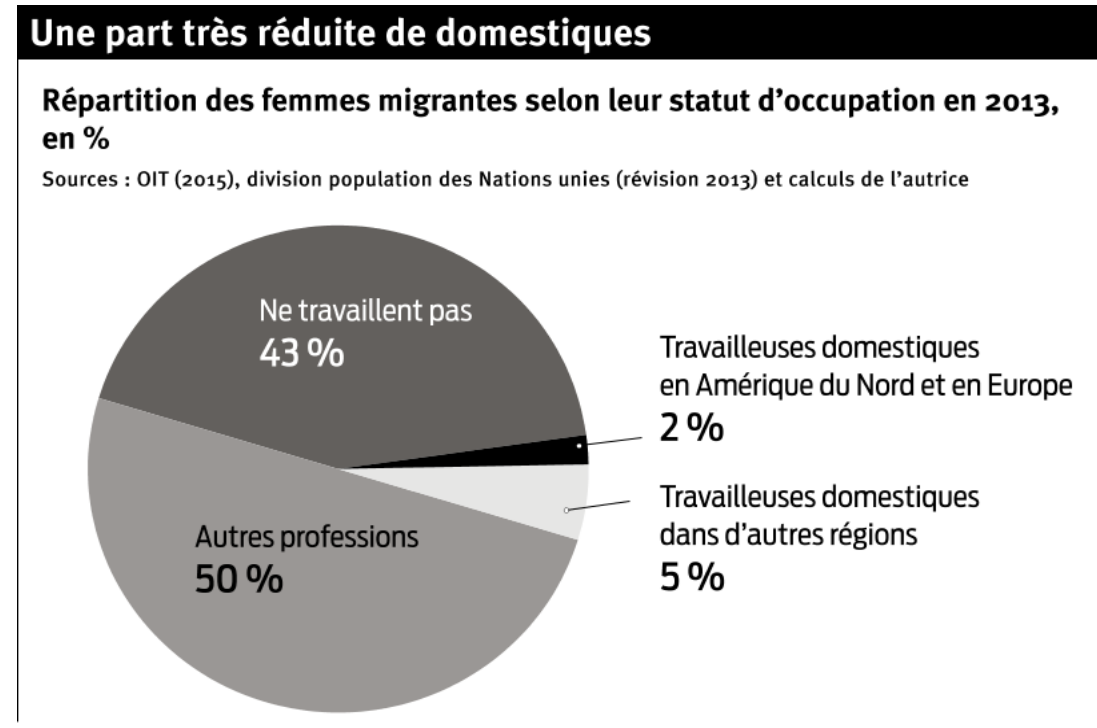

Disposer d'une histoire plausible est une source de biais car elle nous dispense de l'effort de vérifier l'importance numérique des éléments qu'on associe. Ainsi, les tenants de la théorie de la «restructuration économique mondiale » négligent systématiquement les données statistiques sur la migration des femmes. On l'a vu avec Stephen Castles, qui réalise que "la notion de féminisation de la migration ne peut pas être justifiée en termes purement quantitatifs », mais continue à écrire que la « féminisation de la migration » est un fait majeur de la migration internationale. Or, Castles avait été influencé par l'approche néo-marxiste qui voit l'immigration comme la conséquence de l'expansion capitaliste.

La troisième raison de se méfier de la cohérence associative est qu'elle favorise le biais de confirmation (Morewedge \& Kahneman, 2010), c'est-à-dire notre tendance (involontaire) à sélectionner les faits qui confirment l'hypothèse initiale. Par exemple, la théorie de la nouvelle division internationale du travail oriente de nombreuses recherches qualitatives. Elles se concentrent sur l'étude des migrantes originaires des pays en développement travaillant dans le service à la personne dans les pays développés, en négligeant les taux de base. La plupart y découvrent leurs attentes : une division Nord-Sud qu'elles théorisent comme " care drain », " mondialisation du maternage » ou «impérialisme émotionnel ». Ce faisant, elles négligent d'autres phénomènes comme « la féminisation de la migration qualifiée » (Dumitru, 2017).

L'un des exemples est la recherche de Rhacel Parreñas, pour qui «la division internationale du travail est un processus structurel qui détermine la migration des travailleuses domestiques des Philippines ». Jugeant le "capitalisme global» responsable de la "migration et de l'entrée des femmes philippines dans le travail domestique ", elle choisit de conduire des entretiens avec des travailleuses domestiques émigrées des Philippines aux Etats-Unis et en Italie. Elle théorise la "mondialisation du maternage» dans un livre intitulé Les servantes de la mondialisation.

Le biais de confirmation renforce les éventuels stéréotypes et réduit le bénéfice de la recherche qualitative. Par exemple, la moitié des travailleuses domestiques interrogées par Parreñas (2001) avaient accompli des études universitaires. De même, Hochschild (2004) relate sans commenter que l'une des nounous a fait des études d'ingénieure. Cela n'est pas surprenant quand on sait 64 \% les migrantes philippines sont diplômées 
de l'enseignement supérieur (Docquier et al, 2009). Mais le niveau d'éducation ne suscite pas d'intérêt quand on étudie les travailleuses domestiques avec l'idée qu'elles illustrent une division internationale de travail. De fait, la nounou ingénieure servira à théoriser le « care drain » par analogie avec la « fuite des cerveaux » (brain drain) des hommes, tandis que les diplômées philippines reflèteront la «mondialisation du maternage ».

Comment les taux de base auraient pu changer ces résultats, en l'absence du biais de confirmation? Les données de l'Agence de l'Emploi à l'Etranger de Philippines confirment qu'une proportion importante, qui varie entre $27 \%$ et $54 \%$, des femmes émigrent en tant que travailleuses domestiques. Mais les Etats-Unis arrivent à la $20^{\mathrm{e}}$ place en nombre de départs pour ce métier, les principales destinations étant en Asie. En effet, le nombre de femmes qui ont quitté les Philippines pour travailler comme " programmatrices en informatique » aux Etats-Unis est légèrement plus grand que celui des femmes parties comme «aides domestiques »4. D’ailleurs, le statut socioéconomique des migrantes philippines aux Etats-Unis est plutôt élevé : elles sont en majorité diplômées de l'université, gagnent en moyenne plus de 52.000 \$ par an et $42 \%$ d'entre elles travaillent dans les occupations de " management, affaires, science et arts ».

Les données sur l'émigration aux Philippines ne montrent pas seulement que les femmes sont plus nombreuses à partir aux Etats-Unis pour un métiers traditionnellement associés aux hommes (programmeur informatique) qu'aux femmes (travailleuses domestiques). Ces données montrent aussi que les hommes philippins qui émigrent pour le travail domestique sont plus nombreux à choisir des pays européens (Espagne, Italie) que des pays asiatiques, privilégiés par les femmes. A l'inverse, lorsqu'on regarde les statistiques italiennes sur les travailleurs domestiques, on observe que les hommes, quel que soit leur pays d'origine, sont plus vulnérables que les femmes aux changements de lois migratoires. Mais parmi les hommes migrants travaillant dans ce métier, ceux originaires des Philippines sont moins touchés que d'autres groupes, tels les hommes originaires de l'Afrique du Nord ou de l'Europe de l'Est. Toutes ces observations statistiques mériteraient des enquêtes de terrain approfondies.

Pour qui est sensible aux inégalités mondiales et de genre, il vaut mieux se méfier d'histoires plausibles. Certains stéréotypes peuvent être propagés de façon involontaire ou avec de bonnes intentions. C'est le cas de la théorie de la division de travail internationale et genrée qui prédit que la mondialisation capitaliste conduit à la « féminisation de la migration », un phénomène qui inciterait les femmes du Sud à migrer pour travailler dans les services à la personne dans les pays du Nord. Or, les données statistiques ne confirment pas ces prédictions.

Cet article plaide pour une consultation des statistiques disponibles comme préalable à toute recherche qualitative. Non seulement cet effort éviterait la propagation des stéréotypes sur les femmes migrantes, mais il renouvellerait les recherches sur le genre et la migration. Savoir si certaines travailleuses domestiques sont diplômées ou si des hommes travailleurs domestiques perdent plus facilement leur emploi que les femmes sont des exemples de questions qui peuvent éclairer la compréhension des rapports sociaux.

\footnotetext{
${ }^{4}$ L'étude des flux vers les Etats-Unis durnt la période 1992-2010 indique 1495 programmatrices informatiques contre 1444 travailleuses domestiques, voir Dumitru (2018) pour plus de détails.
} 


\section{Biblingraphie}

Acharya A.K., 2010, "Feminization of Migration and Trafficking of Women in Mexico » Revista de Cercetare si Interventie Sociala, vol. 30, pp. 19-38.

Castles S., 1999, "International Migration and the Global Agenda: Reflections on the 1998 UN Technical Symposium." International Migration 37 (1):5-19

Castles S. et Miller, M.J., 2003, The Age of Migration. New York: Guilford

Docquier F., Lowell B. L., Marfouk A., 2009, «A gendered assessment of highly skilled emigration » Population and Development Review, vol. 35, $\mathrm{n}^{\circ}$ 2, pp. 297-322

Dumitru, S., 2017, «Féminisation de la migration qualifiée: les raisons d'une invisibilité » Hommes \& Migrations, $\mathrm{n}^{\circ}$ 1317, pp. 146-153

Dumitru S., 2018, « How neo-Marxism creates bias in gender and migration research: evidence from the Philippines » Ethnic and Racial Studies vol. 41, nº ${ }^{\circ}$, pp. 2790-2808

Fröbel F., Heinrichs J., et Kreye O., 1980, The new international division of labour: structural unemployment in industrialised countries and industrialisation in developing countries. London [etc.]: Cambridge University Press.

Gündüz Z. Y., 2013, "The Feminization of Migration: Care and the New Emotional Imperialism » Monthly Review, vol. 65, ${ }^{\circ} 7, \mathrm{pp} .32-43$

Hochschild, A. R., 2004, « Le nouvel or du monde », trad. de l'anglais par Laurence Bachmann Nouvelles Questions Féministes, vol 23, $\mathrm{n}^{\circ}$ 3, p. 59-74

Kahneman D., 2012, Système 1 Système 2 : Les deux vitesses de la pensée, trad. de l'anglais par Raymond Clarinard, Flammarion, Paris

Morewedge C. K. et Kahneman, D., 2010, “Associative processes in intuitive judgment.” Trends in cognitive sciences vol. $14, \mathrm{n}^{\circ} 10: 435-40$.

OIT, 2015, Global estimates on migrant workers. ILO, Geneva.

Parreñas R. S., 2001, Servants of globalization: Women, Migration, and Domestic Work, Stanford University Press, Stanford.

Passerini L., Lyon D., Capussotti E., et Laliotou I., 2010, Women migrants from East to West: Gender, mobility and belonging in contemporary Europe. New York, Oxford: Berghahn Books.

Rothschild, T.K. « Feminization of Migration », 2009, in Jodi O'Brien (dir.) Encyclopedia of Gender and Society, Thousand Oaks, Sage Publications

Sassen S., 1988, The Mobility of Labor and Capital: A Study in International Investment and Labor Flow. Cambridge, Cambridge University Press.

Yoshimura M., 2007, "Feminization of Labor Migration » in George Ritzer (dir.) Blackwell Encyclopedia of Sociology, Blackwell Publishing.

Zlotnik H., 1990, "International Migration Policies and the Status of Female Migrants » International Migration Review, vol. $24 \mathrm{n}^{\circ} 2$, pp. 372-81. 